\title{
Effect of light sources and curing mode techniques on sorption, solubility and biaxial flexural strength of a composite resin
}

\author{
Andreia Assis CARVALHO', Francine do Couto Lima MOREIRA', Rodrigo Borges FONSECA², Carlos José SOARES ${ }^{3}$, \\ Eduardo Batista FRANCO ${ }^{4}$, João Batista de SOUZA², Lawrence Gonzaga LOPES ${ }^{2}$
}

\author{
1- DDS, MS, School of Dentistry, Federal University of Goiás, Goiânia, GO, Brazil. \\ 2- DDS, MS, PhD, School of Dentistry, Federal University of Goiás, Goiânia, GO, Brazil. \\ 3- DDS, MS, PhD, School of Dentistry, Federal University of Uberlândia, Uberlândia, MG, Brazil. \\ 4- DDS, MS, PhD, Bauru School of Dentistry, University of São Paulo, Bauru, SP, Brazil.
}

Corresponding address: Andreia Assis Carvalho - Universidade Federal de Goiás - Faculdade de Odontologia - Departamento de Prevenção e Reabilitação Oral Praça Universitária, s/n - Faculdade de Odontologia - Setor Universitário - Goiânia - GO - 74605220 - Phone: 556232096050 - Fax: 556235211882 - e-mail: andreia.assiscarvalho@gmail.com

Received: August 13, 2010 - Modification: July 31, 2011 - Accepted: August 25, 2011

\section{ABSTRACT}

\begin{abstract}
A dequate polymerization plays an important role on the longevity of the composite resin restorations. Objectives: The aim of this study was to evaluate the effect of lightcuring units, curing mode techniques and storage media on sorption, solubility and biaxial flexural strength (BFS) of a composite resin. Material and Methods: Two hundred and forty specimens were made of one composite resin (Esthet- $X$ ) in a stainless steel mold ( $2 \mathrm{~mm} X$ $8 \mathrm{~mm} \varnothing)$, and divided into 24 groups $(n=10)$ established according to the 4 study factors: light-curing units: quartz tungsten halogen (QTH) lamp and light-emitting diodes (LED); energy densities: $16 \mathrm{~J} / \mathrm{cm}^{2}$ and $20 \mathrm{~J} / \mathrm{cm}^{2}$; curing modes: conventional (CM) and pulsedelay (PD); and permeants: deionized water and $75 \%$ ethanol for 28 days. Sorption and solubility tests were performed according to ISO 4049:2000 specifications. All specimens were then tested for BFS according to ASTM F394-78 specification. Data were analyzed by three-way ANOVA followed by Tukey, Kruskal-Wallis and Mann-Whitney tests $(\alpha=0.05)$. Results: In general, no significant differences were found regarding sorption, solubility or BFS means for the light-curing units and curing modes ( $p>0.05)$. Only LED unit using $16 \mathrm{~J} / \mathrm{cm}^{2}$ and PD using $10 \mathrm{~s}$ produced higher sorption and solubility values than QTH. Otherwise, using CM $\left(16 \mathrm{~J} / \mathrm{cm}^{2}\right)$, LED produced lower values of BFS than QTH $(p<0.05)$. $75 \%$ ethanol permeant produced higher values of sorption and solubility and lower values of BFS than water $(p<0.05)$. Conclusion: Ethanol storage media produced more damage on composite resin than water. In general the LED and QTH curing units using 16 and 20 $\mathrm{J} / \mathrm{cm}^{2}$ by $\mathrm{CM}$ and PD curing modes produced no influence on the sorption, solubility or BFS of the tested resin.
\end{abstract}

Key words: Composite resins. Solubility. Solvents. Mechanical phenomena.

\section{INTRODUCTION}

Light-cured composite resins are being more used specially because they are aesthetically advantageous and enable to be polymerized by physical and chemical activators ${ }^{8,30}$. However, the crucial point to be reached during restorative dentistry procedures with composite resins is to obtain satisfactory restorations with an adequate light activation technique ${ }^{24}$.
Composites consist mainly of filler particles and a resin matrix based on different monomers ${ }^{6}$. Currently, the most widely marketed composite resins are based on the polymerization of bisphenol A glycol dimethacrylate (Bis-GMA) and urethane dimethacrylate (UDMA) ${ }^{25}$. The curing process occurs in the organic matrix where there is a monomer-polymer conversion through an activation mechanism $^{8}$. However, an insufficient degree of conversion directly affects the physical properties 
and chemical stability of material ${ }^{8}$. Polymers used in the composition of composite resins may absorb water and chemicals from the oral environment (sorption) and may release some of their own components (solubility) ${ }^{1,12}$. Restorations are subject to tension due to chewing when an excessive or continue non-functional load is applied, internal stresses and strain are generated which can cause the material failure ${ }^{27}$.

In this context, the adequate polymerization plays an important role on the longevity of composite resin restorations. This procedure requires sufficient light energy intensity and an adequate wavelength in order to activate the photoinitiator within these materials, which will react with the reducer agent to form free radicals and initiate the polymerization process ${ }^{24}$. Variables such as adequate light intensity, correct wavelength and energy density (power density $x$ exposure time) are essential for achieving the proper depth of cure ${ }^{1}$. Different types of light curing units have been proposed for the polymerization of light activated resinous materials including conventional quartz tungsten halogen (QTH) lamp, plasma arc, argon laser and light-emitting diodes (LED). All these devices emit blue light in the spectrum of the absorption of camphorquinone, which is the photoinitiator generally found in most resinous material ${ }^{9}$. The QTH lights can achieve satisfactory results, but they present certain limitations such as shorter durability of the lamp and heat production ${ }^{9}$. LED have been recognized as a promising technology for polymerization of resin-based materials because all the light emitted is within the spectrum of maximum absorption of camphorquinone at $468 \mathrm{~nm}^{1,13}$.

Different curing regimes can exert an influence on the resistance of the union, hardness and crosslinking density ${ }^{7}$. When high power density is applied, more photoinitiator molecules are activated at the same time and consequently acceleration occurs immediately upon light exposure, thereby generating inner stresses that are confined into composite, dental structure and interfaces ${ }^{21}$.

In the pulse-delay (PD) polymerization technique there is a decrease in shrinkage and stress ${ }^{30}$. In this technique, there is a short period (about 1-3 min) between initial exposition and final cure ${ }^{10}$. The interval between both pulses, an initial pulse with low energy density and a final pulse with high light irradiation, increases the composite resin pregel phase improving its flowability and relieving the stress generate by composite shrinkage. Thus, a better marginal seal is obtained ${ }^{17,30}$. It has been hypothesized that low power densities, characterized by reduced power density in the initial seconds, may generate a small number of free radicals. Then, a more linear polymeric structure is obtained, with lower crosslinking density, which negatively affects the material's chemical and physical properties ${ }^{28}$. A previous study ${ }^{4}$ concluded that marginal integrity of restorations and the composite "plasticizing effect" are negatively affected when a PD technique is used.

The aim of this study was to investigate the effect of different light sources, PD curing protocols and storage media on sorption, solubility and biaxial flexural strength (BFS) of a composite resin. The null hypotheses tested were: (1) QTH and LED curing light units using different energy densities produce no influence on sorption, solubility and BFS; (2) QTH and LED curing light units using different curing techniques produce no influence on sorption, solubility and BFS; (3) storage procedure in water and ethanol produces no influence on sorption, solubility and BFS.

\section{MATERIAL AND METHODS}

\section{Preparation of specimens}

A nanohybrid composite resin (Esthet-X, Dentsply, Rio de Janeiro, RJ, Brazil) (Batch \#071116), A2 shade, was used to perform the sorption and solubility test and biaxial piston-onring flexural strength test. The specimens were made in compliance with ISO 4049:2000 standard specifications ${ }^{14}$. Specimens were made using a stainless steel mold with $2 \mathrm{~mm}$ in thick and $8 \mathrm{~mm}$ in diameter. Two hundred and forty specimens were divided into 24 groups $(n=10)$ established according to the 4 study factors:

1- light-curing units: QTH lamp (Variable Intensity Polymerizer, Bisco Inc., Shaumburg, IL, USA) and LED (Ultra Blue IS, DMC, São Carlos, SP, Brazil), 2- energy densities: $16 \mathrm{~J} / \mathrm{cm}^{2}$ and $20 \mathrm{~J} / \mathrm{cm}^{2}$, 3- curing modes: conventional mode (CM), pulsedelay using $3 \mathrm{~s}$ (PD3s) and pulse-delay using $10 \mathrm{~s}$ (PD10s); 4- storage media (for 28 days): deionized water and $75 \%$ ethanol (Figure 1 ).

The light irradiance for each curing mode was checked with the in-built radiometer prior to use to ensure consistency of light output. A single increment of composite material was placed into a stainless steel mold and confined between two opposing polyester strips (K-Dent, Quimidrol, Joinville, SC, Brazil). The material was irradiated according each protocol. The specimens were then removed from the mold and excesses were eliminated with a scalpel blade (Free-Bac, Wuxi Xinda Medical Device Co. Ltd., Wuxi City, Jiangsu, China).

\section{Sorption and solubility tests}

The sorption and solubility tests were performed in compliance with ISO 4049:200014 standard specifications, except for the specimen dimensions, types of permeant and storage time. The specimen 
was placed individually into an open glass bottle of $20 \mathrm{~mm}$ (Verallia; Saint-Gobain Vidros S.A., São Paulo, SP, Brazil) inserted into the desiccators (Vidrolabor; Vidrolabor Ind. Com., São Paulo, SP, Brazil) containing freshly dried white silica, batch \#0506198, (Vetec, Vetec Química Fina Ltda, Rio de Janeiro, RJ, Brazil), and maintained for $22 \mathrm{~h}$ at $37 \pm 1^{\circ} \mathrm{C}$ in a vacuum oven (Model $440 \mathrm{D}$, LF Equipamentos, São Paulo, SP, Brazil). Thereafter, desiccators containing the specimens were removed from the oven and placed on a bench for $2 \mathrm{~h}$, at a temperature of $23 \pm 1^{\circ} \mathrm{C}$, completing a cycle of 24 h. Specimens were weighed daily on an analytical scale accurate to $0.001 \mathrm{mg}$ (Marte AY220, Marte Balanças e Aparelhos de Precisão Ltda, Santa Rita do Sapucaí, MG, Brazil). The complete cycle was repeated until a constant mass (M1) was obtained, that is, until the mass loss of each specimen was not more than $0.1 \mathrm{mg}$ per $24 \mathrm{~h}$ cycle. Afterwards, the specimens were returned to their respective labeled bottles, and $15 \mathrm{~mL}$ of either deionized water or $75 \%$ ethanol were added with manual pipettes. The bottles were capped, replaced in the oven and kept at $37 \pm 1^{\circ} \mathrm{C}$ for 28 days.

After this period, all bottles were removed from the oven and kept at room temperature, $23 \pm 1^{\circ} \mathrm{C}$ for $2 \mathrm{~h}$. The specimens were removed from the bottles, dried with absorbent paper for $15 \mathrm{~s}$ and left in a sterile bucket (Duflex, SSWhite, Rio de Janeiro, RJ, Brazil) for $1 \mathrm{~min}$. Specimens were weighed to obtain M2. Then the specimens were returned to the desiccators until they reached a constant weight (M3) using the same procedure described to obtain M1.

The values for water sorption and solubility were calculated in $\mu \mathrm{g} / \mathrm{mm}^{3}$ using the following equations: Wsp $=(M 2-M 3) / \mathrm{V} ; \mathrm{Wsl}=(\mathrm{M} 1-\mathrm{M} 3) / \mathrm{V}$, where $\mathrm{Wsp}$ was the sorption, Wsl was the solubility, M1 was a initial mass of the sample in $\mathrm{mg}, \mathrm{M} 2$ was a mass of the specimen after immersion into storage media in $\mathrm{mg}$, M3 was a final mass of the specimen recorded after to evaporation of the water or ethanol in $\mathrm{mg}$, and $\checkmark$ was a volume of the specimen in $\mathrm{mm}^{3}$.

\section{Biaxial flexural strength test}

After sorption and solubility tests, all specimens were submitted to a biaxial piston-on-ring flexural strength test. The specimen was positioned on a circular metallic device with $3 \mathrm{~mm}$ in radius that contain 3 symmetrically spaced steel spheres.

\begin{tabular}{|c|c|c|c|c|c|}
\hline Light sources & Power density $\left(\mathrm{J} / \mathrm{cm}^{2}\right)$ & Curing modes & & g profi & \\
\hline \multirow{6}{*}{ QTH } & \multirow{3}{*}{16} & Conventional (CM) & $27 \mathrm{~s}-600 \mathrm{~mW} / \mathrm{cm}^{2}$ & & \\
\hline & & Pulse delay (PD3 s) & $3 \mathrm{~s}-300 \mathrm{~mW} / \mathrm{cm}^{2}$ & $2 \min$ & $25 \mathrm{~s}-600 \mathrm{~mW} / \mathrm{cm}^{2}$ \\
\hline & & Pulse delay (PD10 s) & $10 \mathrm{~s}-300 \mathrm{~mW} / \mathrm{cm}^{2}$ & $2 \min$ & $22 \mathrm{~s}-600 \mathrm{~mW} / \mathrm{cm}^{2}$ \\
\hline & \multirow{3}{*}{20} & Conventional (CM) & $34 \mathrm{~s}-600 \mathrm{~mW} / \mathrm{cm}^{2}$ & $\overline{-}$ & $\overline{-}$ \\
\hline & & Pulse delay (PD3 s) & $3 \mathrm{~s}-300 \mathrm{~mW} / \mathrm{cm}^{2}$ & $2 \min$ & $32 \mathrm{~s}-600 \mathrm{~mW} / \mathrm{cm}^{2}$ \\
\hline & & Pulse delay (PD10 s) & $10 \mathrm{~s}-300 \mathrm{~mW} / \mathrm{cm}^{2}$ & $2 \min$ & $28 \mathrm{~s}-600 \mathrm{~mW} / \mathrm{cm}^{2}$ \\
\hline \multirow{6}{*}{ LED } & \multirow{3}{*}{16} & Conventional (CM) & $27 \mathrm{~s}-600 \mathrm{~mW} / \mathrm{cm}^{2}$ & & \\
\hline & & Pulse delay (PD3 s) & $3 \mathrm{~s}-300 \mathrm{~mW} / \mathrm{cm}^{2}$ & $2 \min$ & $25 \mathrm{~s}-600 \mathrm{~mW} / \mathrm{cm}^{2}$ \\
\hline & & Pulse delay (PD10 s) & $10 \mathrm{~s}-300 \mathrm{~mW} / \mathrm{cm}^{2}$ & $2 \min$ & $22 \mathrm{~s}-600 \mathrm{~mW} / \mathrm{cm}^{2}$ \\
\hline & \multirow{3}{*}{20} & Conventional (CM) & $34 \mathrm{~s}-600 \mathrm{~mW} / \mathrm{cm}^{2}$ & & \\
\hline & & Pulse delay (PD3 s) & $3 \mathrm{~s}-300 \mathrm{~mW} / \mathrm{cm}^{2}$ & $2 \min$ & $32 \mathrm{~s}-600 \mathrm{~mW} / \mathrm{cm}^{2}$ \\
\hline & & Pulse delay (PD10 s) & $10 \mathrm{~s}-300 \mathrm{~mW} / \mathrm{cm}^{2}$ & $2 \min$ & $28 \mathrm{~s}-600 \mathrm{~mW} / \mathrm{cm}^{2}$ \\
\hline
\end{tabular}

Figure 1- Details of the various light curing modes evaluated.

Table 1- Means, standard deviations $( \pm S D$ ) for comparison between light source and permeant for the measurement of sorption and solubility at $16 \mathrm{~J} / \mathrm{cm}^{2}$

\begin{tabular}{ccccccc}
\hline $\begin{array}{c}\text { Interation light } \\
\text { source + permeant }\end{array}$ & \multicolumn{3}{c}{ Sorption } & & \multicolumn{3}{c}{ Solubility } \\
& CM & PD3 s & PD10 s & CM & PD3 s & PD10 s \\
\hline QTH-water & $9.97^{\mathrm{a}}(0.95)$ & $11.29^{\mathrm{a}}(2.66)$ & $10.76^{\mathrm{a}}(1.88)$ & $1.71^{\mathrm{a}}(0.78)$ & $3.08^{\mathrm{a}}(2.40)$ & $1.68^{\mathrm{a}}(1.56)$ \\
\hline LED-water & $10.02^{\mathrm{a}}(1.39)$ & $12.08^{\mathrm{a}}(1.67)$ & $11.22^{\mathrm{a}}(1.48)$ & $1.68^{\mathrm{a}}(1.48)$ & $2.04^{\mathrm{a}}(1.64)$ & $3.01^{\mathrm{a}}(1.45)$ \\
\hline QTH-ethanol & $19.02^{\mathrm{b}}(2.21)$ & $18.77^{\mathrm{b}}(0.94)$ & $18.39^{\mathrm{b}}(2.60)$ & $6.75^{\mathrm{b}}(2.33)$ & $6.33^{\mathrm{b}}(2.07)$ & $6.74^{\mathrm{b}}(1.47)$ \\
\hline LED-ethanol & $19.98^{\mathrm{b}}(1.46)$ & $20.23^{\mathrm{b}}(1.96)$ & $21.54^{\mathrm{c}}(2.56)$ & $6.86^{\mathrm{b}}(1.59)$ & $8.47^{\mathrm{b}}(1.86)$ & $10.70^{\mathrm{c}}(2.22)$ \\
\hline
\end{tabular}

Values in the column with same superscript lower caser letter denote no statistical differences $(p>0.05)$ 
Table 2- Means, standard deviations $( \pm S D$ ) for comparison between light source and permeant for the measurement of sorption and solubility at $20 \mathrm{~J} / \mathrm{cm}^{2}$

\begin{tabular}{ccccccc}
\hline $\begin{array}{c}\text { Interation light } \\
\text { source + permeant }\end{array}$ & \multicolumn{3}{c}{ Sorption } & \multicolumn{3}{c}{ Solubility } \\
& CM & PD3 s & PD10 s & CM & PD3 s & PD10 s \\
\hline QTH-water & $9.96^{\mathrm{a}}( \pm 1.53)$ & $10.49^{\mathrm{a}}( \pm 2.34)$ & $12.15^{\mathrm{a}}( \pm 1.71)$ & $0.36^{\mathrm{a}}( \pm 0.61)$ & $1.41^{\mathrm{a}}( \pm 1.83)$ & $1.78^{\mathrm{a}}( \pm 1.28)$ \\
LED-water & $10.24^{\mathrm{a}}( \pm 1.77)$ & $11.40^{\mathrm{a}}( \pm 2.74)$ & $10.84^{\mathrm{a}}( \pm 0.94)$ & $0.01^{\mathrm{a}}( \pm 0.00)$ & $3.02^{\mathrm{a}}( \pm 2.71)$ & $1.63^{\mathrm{a}}( \pm 1.49)$ \\
\hline QTH-ethanol & $16.58^{\mathrm{b}}( \pm 2.04)$ & $18.93^{\mathrm{b}}( \pm 2.20)$ & $19.05^{\mathrm{b}}( \pm 1.54)$ & $4.95^{\mathrm{b}}( \pm 2.16)$ & $4.78^{\mathrm{b}}( \pm 1.36)$ & $6.45^{\mathrm{b}}( \pm 1.88)$ \\
\hline LED-ethanol & $17.89^{\mathrm{b}}( \pm 1.19)$ & $20.52^{\mathrm{b}}( \pm 2.53)$ & $19.59^{\mathrm{b}}( \pm 2.15)$ & $4.95^{\mathrm{b}}( \pm 1.53)$ & $7.30^{\mathrm{b}}( \pm 2.63)$ & $6.55^{\mathrm{b}}( \pm 2.54)$ \\
\hline
\end{tabular}

Values in the column with same superscript lower caser letter denote no statistical differences $(p>0.05)$

Table 3- Means, standard deviations ( $\pm S D$ ) for biaxial flexural strength at $16 \mathrm{~J} / \mathrm{cm}^{2}$

\begin{tabular}{ccccccc}
\hline & \multicolumn{5}{c}{ QTH } & \multicolumn{4}{c}{ LED } \\
& CM & PD3 s & PD10 s & CM & PD3 s & PD10 s \\
\hline water & $128.52^{\mathrm{A}}$ & $107.09^{\mathrm{B}}$ & $114.28^{\mathrm{A}, \mathrm{B}}$ & $106.62^{\mathrm{B}}$ & $119.69^{\mathrm{A}, \mathrm{B}}$ & $122.15^{\mathrm{A}, \mathrm{B}}$ \\
& $( \pm 17.81)$ & $( \pm 21.17)$ & $( \pm 9.74)$ & $( \pm 24.97)$ & $( \pm 18.67)$ & $( \pm 15.14)$ \\
ethanol & $63.09^{\mathrm{C}}( \pm 7.67)$ & $56.43^{\mathrm{C}}( \pm 11.29)$ & $51.76^{\mathrm{C}}( \pm 5.88)$ & $59.88^{\mathrm{C}}( \pm 9.07)$ & $50.69^{\mathrm{C}}( \pm 6.85)$ & $46.70^{\mathrm{C}}( \pm 5.98)$ \\
\hline
\end{tabular}

Values with same superscript capital letter (A, B or C) denote no statistical differences $(p>0.05)$

Table 4- Means, standard deviations $( \pm S D)$ for biaxial flexural strength at $20 \mathrm{~J} / \mathrm{cm}^{2}$

\begin{tabular}{ccccccc}
\hline & \multicolumn{3}{c}{ QTH } & \multicolumn{3}{c}{ LED } \\
& CM & PD3 s & PD10 s & CM & PD3 s & PD10 s \\
\hline water & $121.32^{\mathrm{Aa}}$ & $111.98^{\mathrm{Aa}}$ & $130.33^{\mathrm{Aa}}$ & $118.09^{\mathrm{Aa}}$ & $118.44^{\mathrm{Aa}}$ & $111.26^{\mathrm{Aa}}$ \\
& $( \pm 25.40)$ & $( \pm 23.79)$ & $( \pm 16.39)$ & $( \pm 28.90)$ & $( \pm 9.20)$ & $( \pm 15.14)$ \\
\multirow{2}{*}{ ethanol } & $68.36^{\mathrm{Ba}}$ & $60.13^{\mathrm{Ba}}$ & $58.21^{\mathrm{Ba}}$ & $58.30^{\mathrm{Ba}}$ & $55.85^{\mathrm{Ba}}$ & $55.80^{\mathrm{Ba}}$ \\
& $( \pm 11.44)$ & $( \pm 9.36)$ & $( \pm 10.13)$ & $( \pm 12.99)$ & $( \pm 6.94)$ & $( \pm 7.84)$ \\
\hline
\end{tabular}

Values in the same column with same superscript minute letter (A or B) denote no statistical differences ( $p>0.05$ ) Values in the same line with same superscript capital letter (a or b) denote no statistical differences $(p>0.05)$

Each specimen was placed concentrically on the supporting spheres to ensure that the load was applied at the center of the specimen. A steel piston of $2 \mathrm{~mm}$ in diameter was attached to a universal testing machine (EMIC, DL2000, São José dos Pinhais, PR, Brazil) and the test was performed at a crosshead speed of $0.5 \mathrm{~mm} / \mathrm{min}$ using a $50 \mathrm{Kgf}$ load cell until specimen failure. The BFS was calculated according to the ASTM F394-78 specifications as follows ${ }^{2}$ :

$S=-0.2387 P(X-Y) / d^{2}$; where $S$ is the flexural strength in $\mathrm{MPa}, \mathrm{P}$ is the load at failure in $\mathrm{N}$, and $\mathrm{d}$ is the specimen thickness in $\mathrm{mm}$ of the failure area. $X$ and $Y$ were determined as follows: $X=(1+v)$ In $(B / C)^{2}+[(1-v) / 2](B / C)^{2}$ and $Y=(1+v)[1+\ln$ $\left.(A / C)^{2}\right]+(1-v)(A / C)^{2}$, where $v$ is Poisson's ratio of the composite resin $(0.24)^{20}, A$ is the radius of the support circle, $B$ is the radius of the tip of the piston, and $C$ is the radius of the specimen.

Data obtained from the sorption, solubility and BFS tests were subjected to three-way analysis of variance and Tukey's tests for parametric values, and Kruskal-Wallis and Mann-Whitney tests for non-parametric values $(a=0.05)$. Pearson's correlation was used to verify the correlation among the sorption, solubility and BFS. All tests were performed using the SPSS 17.0 for Windows statistics software (SPSS Inc., Chicago, IL, USA).

\section{RESULTS}

The non-parametric data were analyzed in terms of the average position. However, to facilitate understanding, the data presented in the tables are the means of the groups with their respective standard deviations ( \pm s.d.). The results for sorption and solubility at energy densities of $16 \mathrm{~J} / \mathrm{cm}^{2}$ and $20 \mathrm{~J} / \mathrm{cm}^{2}$ are shown in Tables 1 and 2, respectively. In general, no significant difference was found between the light sources and between the curing modes tested in this study $(p>0.05)$. However, in the interaction between permeant and light source 
Table 5- Pearson's correlation coefficient

\begin{tabular}{cccc}
\hline & Sorption & Solubility & BFS \\
\hline Sorption & --- & $+0.852^{*}$ & $-0.840^{*}$ \\
Solubility & $+0.852^{*}$ & --- & $-0.781^{*}$ \\
BFS & $-0.840^{*}$ & $-0.781^{*}$ & --- \\
\hline
\end{tabular}

* significant correlation $(p<0.05)$.

with a density of $16 \mathrm{~J} / \mathrm{cm}^{2}$, it was seen that the specimens light-cured with the LED unit device in PD10s and stored in ethanol led to higher values of sorption and solubility than those light-cured with QTH $(p<0.05)$. Ethanol showed higher sorption and solubility than water irrespective of curing unit or curing method $(p<0.05)$.

In the BFS there were interactions between light sources, curing modes and permeants at an energy density of $16 \mathrm{~J} / \mathrm{cm}^{2}(p<0.05)$ (Table 3), which did not occur at a density of $20 \mathrm{~J} / \mathrm{cm}^{2}$, where there were significant differences only between the permeants (water and ethanol) (Table 4).

The correlation among sorption, solubility and BFS was performed by Pearson's correlation coefficient test. It was found that sorption and solubility data had a positive correlation. Otherwise, BFS were negatively correlated with sorption and solubility (Table 5).

\section{DISCUSSION}

Successful composite resin restoration depends on the association of low rate shrinkage, good flowability, appropriate cure and satisfactory mechanical properties ${ }^{13}$. LED sources seem to be a promising technology for polymerization of dental resin materials, but its application in composite curing remains controversial ${ }^{19}$. In this study, QTH lamp was adopted as a control in order to compare its performance with LED. LED units produce a narrow band of wavelengths (450-490 nm), conveniently situated within the absorption spectrum of camphorquinone, which is the photoinitiator present in the most of light-activated dental materials ${ }^{13}$. In general, the LED unit showed similar behavior to the QTH unit, suggesting that similar polymerization quality was reached by the equipment, except when LED using $16 \mathrm{~J} / \mathrm{cm}^{2}$ in PD10 s intensity produced higher values of sorption and solubility than QTH $(p<0.05)$. LED curing unit using $C M$ at $16 \mathrm{~J} / \mathrm{cm}^{2}$ produced lower values of BFS than QTH used with the same protocol $(p<0.05)$. This fact could be associated with the greater heat generated by the QTH unit, which may speed up the polymer chain induction process in composite, increasing the mobility of molecules during the reaction and allowing more monomers to react before the curing process ends ${ }^{13}$. The polymerization does not stop right after the photoactivation period, and heat contributes for this post-activation polymerization ${ }^{19}$. This could be further explained by the absorbance of camphorquinone. Although camphorquinone presents its maximum absorbance at $468 \mathrm{~nm}$, it has an absorption band (380-510 nm) that is coincident with the light band emitted by QTH lamps resulting in more camphorquinone molecules being activated $^{22}$. Consequently, the crosslinked polymer was probably lower in these LED subgroups than in the corresponding QTH subgroups and more susceptible to softening in solvents.

The size of the specimens used in this study differed from the ISO $4049: 2000^{14}$, as the standard dictates that they must be $15 \mathrm{~mm}$ in diameter. This contrasts with the diameter of the light guide tip of the curing units, which are 8 to $10 \mathrm{~mm}$ in diameter, and which would limit the uniform irradiation throughout the circumference of the specimen.

Traditionally, the manufacturers have recommended high light intensity or power density to provide a higher degree of monomer conversion into polymer, thereby improving the mechanical properties of composite resin ${ }^{17,18}$. High power density used for short light exposures led to a lower degree of cure and lower flexural strength and modulus than when the composite was cured with intermediary power densities for longer exposures ${ }^{23}$. In some studies ${ }^{18,28}$ that used $24 \mathrm{~J} / \mathrm{cm}^{2}$ of energy density, the curing modes tested presented similar behavior. It has been speculated that this occurred as a result of the formation of a densely crosslinked polymer network because an adequate energy density had been used ${ }^{18}$. The same finding was seen in this study in the groups that used $20 \mathrm{~J} / \mathrm{cm}^{2}$, where there were no significant differences between the subgroups studied. The subgroups that used $16 \mathrm{~J} /$ $\mathrm{cm}^{2}$ presented different values of sorption, solubility and BFS storage in ethanol for the QTH and LED units $(p<0.05)$. Consequently, the first hypothesis of this study was partially rejected.

The use of low initial intensity followed by high intensity light with an interval between them seems to create a uniform polymerization of the composite resin, providing its best adaptation to cavity walls and possibly the least polymerization contraction stress ${ }^{10,17,30}$. In the delay period, little amount of free radicals and double bond conversion are produced and the composite resin has more time to molecular rearrangement and stress relief ${ }^{10}$. In the PD cure, there was reduced gap formation without any mechanical properties being compromised, which can be considered as an indicative that composite has the same quantity of remaining double bonds ${ }^{3}$. The use of slow-cure methods (pulse curing modes) in combination with the interval between two irradiations seem not to interfere with solvent sorption and could be useful 
for adhesive composite restorations ${ }^{18}$. In this study, there were no significant differences among the curing modes in terms of sorption, solubility and BFS ( $p>0.05$ ) except for QTH comparing the CM and PD3s at $16 \mathrm{~J} / \mathrm{cm}^{2}$, where PD3s produced lower values of BFS than the CM $(p<0.05)$. Therefore, the second hypothesis proposed in the study was accepted. However, this result was different from those of other studies 3,29 , where the PD mode resulted in a linear polymer structure that was less crosslinked, thereby increasing the susceptibility of polymers to softening in ethanol. Some studies ${ }^{3,16}$ have shown a significant reduction in mechanical properties, such as flexural modulus and hardness, when composites were photo-cured by PD mode and submitted to solvent action. However, based on the methodology used in this study and the obtained results, the curing modes presented similar behavior. According to Lopes, et al. ${ }^{18}$ (2009), this could have occurred because another study $^{3}$ used unfilled resins for their tests instead of filled composite resin. Another explanation for the difference between the results of this study and that where the photo-cured composites using the PD mode were more susceptible to softening in solvent could be attributed to the type of tests applied to assess the amount of crosslinking, such as hardness deterioration, degree of conversion and remaining double bonds, which only make an indirect assessment of the quality of the polymer ${ }^{3}$.

The effect of the different solvents was clearly significant in all groups; ethanol resulted in more solubility, sorption and lower BFS than water media. Therefore, the third hypothesis was rejected. In the ISO 4049:2000 specifications ${ }^{14}$, the storage time is 7 days, but in the present study the storage time of 28 days in the same way as established for other studies ${ }^{12,25}$ in which most of the composites studied reached saturation within 7-60 days. The amount of solvent uptake by the polymer is determined by differences in solubility parameters between the polymer and the permeant ${ }^{19}$. Water or solvent uptake into the resin phase of CR causes two opposing processes: the solvent will extract unreacted components, mainly monomer, thereby resulting in shrinkage, loss of weight and reduction in mechanical properties; conversely, solvent uptake leads to a swelling of the composite resin and an increase in weight ${ }^{25}$. These phenomena of sorption and solubility may serve as precursors to a variety of chemical and physical processes which create biological concerns and produce deleterious effects on the structure and function of the polymer material ${ }^{12}$.

It is desirable that composites have all monomers converted into polymers during the polymerization reaction and remain stable for long time ${ }^{11}$. It may be assumed that, whereas the increase in the degree of conversion reduced the solubility because the amount of unreacted monomers available for leaching out was lower due to the high percentage of reacted aliphatic $\mathrm{C}=\mathrm{C}$ bonds from the dimethacrylate monomers ${ }^{6}$. Most dental polymers present ionic functional groups with water affinity, which in turn optimizes their hydrophilicity ${ }^{11}$. The susceptibility for more linear or less crosslinked polymer to softening in solvents may be explained by the solvent-polymer interaction and, consequently, by Hoy's solubility parameter for polar forces ${ }^{16}$. Solvents that can form strong secondary bonds with the polymer chains can penetrate and replace the interchange secondary bonds, and thereby pull apart and dissolve linear and branched polymers ${ }^{25}$. Distilled water, a solvent indicated in ISO specifications ${ }^{14}$ for resin-based filling materials, simulates the wet intraoral environment provided by saliva and water. However, intraoral conditions are clearly more complex than those achieved with distilled water in the laboratory ${ }^{25}$. According to the US Food and Drug Administration Guidelines, a 75\% ethanol-water solution is a clinically relevant food/ oral simulating liquid ${ }^{25,26}$. The $75 \%$ ethanol solvent is the most frequently used to simulate accelerated ageing of restorations as it has a solubility parameter matching that of BisGMA ${ }^{12,25}$ When ethanol penetrates the polymer network, it causes the structure swell, thereby allowing for the release of uncured monomers and causing the dissolution of linear polymer chains ${ }^{12}$. This expansion is facilitated when crosslink density is low, based on the fact that more space and pathways are available for solvent molecules to diffuse within the structure ${ }^{8}$. For this reason, water and $75 \%$ ethanol were used in this experiment.

The properties of composite resins are affected by the degree of conversion, but an analysis of the amount of crosslinking in the resins may provide a closer correlation to mechanical properties ${ }^{12}$. Water sorption may deteriorate polymer mechanical properties and because of this the resin specimens were submitted to a mechanical test after the sorption and solubility tests. Tensile strength is generally considered to be the most meaningful property of these brittle materials in assessing the potential for failure of dental restorations ${ }^{5}$. Flexural strength can be measured using a threepoint bending flexural test, a four-point bending flexural test or a biaxial flexure test; the drawback of the three-point and four-point flexural tests is the inherent sensitivity to flaws and defect near specimen edges ${ }^{15}$. As Ban and Anusavice ${ }^{5}$ (1990) related, the biaxial flexure test is recognized as a reliable technique since the maximum tensile stress occurs within the central loading area and edge failures are eliminated.

In the evaluation of the correlation among the 
variables (sorption, solubility and BFS), it was possible to see how much higher the sorption and solubility were and how much lower the BFS $(p<0.05)$ was. The degree of conversion and crosslink density can influence the chemical degradation that is usually caused by oxidation and hydrolysis processes ${ }^{12}$, and consequently reduce the physical and mechanical properties. Thus, polymers with lower crosslink densities are expected to undergo more softening in solvents ${ }^{29}$, which results in greater sorption and solubility values but lower BFS values.

\section{CONCLUSION}

Within the limitations of this in vitro study methodology, it was concluded that, in general, the light sources (QTH and LED) and curing modes (CM and PD) did not influence the sorption, solubility or BFS of the tested resin. However, the different solvents (water and ethanol) did influence its sorption, solubility and BFS behavior.

\section{ACKNOWLEDGMENTS}

The authors would like to thank Núbia Miranda Ferreira (Dentsply) for supplying materials and Paulo César Freitas Santos Filho for his assistance at the Federal University of Uberlândia's laboratory.

\section{REFERENCES}

1- Aguiar FH, Braceiro A, Lima DA, Ambrosano GM, Lovadino JR. Effect of light curing modes and light curing time on the microhardness of a hybrid composite resin. J Contemp Dent Pract. 2007;8(6):1-8.

2- American Society for Testing and Materials. ASTM F394-78: Test method for biaxial flexure strength (Modulus of Rupture) of ceramic substrates. West Conshohocken: ASTM International; 1996.

3- Asmussen E, Peutzfeldt A. Influence of pulse-delay curing on softening of polymer structures. J Dent Res. 2001;80(6):1570-3. 4- Asmussen E, Peutzfeldt A. Polymerization contraction of resin composite vs. energy and power density of light-cure. Eur J Oral Sci. 2005;113:417-21.

5- Ban S, Anusavice KJ. Influence of test method on failure stress of brittle dental materials. J Dent Res. 1990;69(12):1791-9.

6- Berger SB, Palialol ARM, Cavalli V, Giannini M. Characterization of water sorption, solubility and filler particles of light-cured composite resins. Braz Dent J. 2009;20(4):314-8.

7- Brandt WC, Moraes RR, Correr-Sobrinho L, Sinhoreti AC, Consani S. Effect of different photo-activation methods on push out force, hardness and cross-link density of resin composite restorations. Dent Mater. 2008;24(6):846-50.

8- Caughman WF, Rueggeberg FA, Curtis JW Jr. Clinical guidelines for photocuring restorative resins. J Am Dent Assoc. 1995; 126(9):1281-6.

9- Correr AB, Sinhoreti MAC, Correr-Sobrinho L, Tango RN, Consani S, Schneider LFJ. Effect of exposure time vs. irradiance on knoop hardness of dental composites. Mat Res. 2006;9(3):275-80.

10- Dall'Magro E, Correr AB, Costa AR, Correr GM, Consani $R L$, Correr-Sobrinho $L$, et al. Effect of different photoactivation techniques on the bond strength of a dental composite. Braz Dent J. $2010 ; 21(3): 220-4$.
11- Fabre HSC, Fabre S, Cefaly DF, Carrilho MRO, Garcia FCP, Wang L. Water sorption and solubility of dentin bonding agents light-cured with different light sources. J Dent. 2007;35:253-8.

12- Ferracane JL. Hygroscopic and hydrolytic effects in dental polymer networks. Dent Mater. 2006;22(3):211-22.

13- Franco EB, Santos PA, Mondelli RFL. The effect of different light-curing units on tensile strength and microhardness of a composite resin. J Appl Oral Sci. 2007;15(6):470-4.

14- International Organization for Standardization. ISO 4049: Dentistry polymer - based filling, restorative and luting materials. Geneva: The Organization; 2000.

15- Karakoca S, Yilmaz $\mathrm{H}$. Influence of surface treatments on surface roughness, phase transformation, and biaxial flexural strength of Y-TZP ceramics. J Biomed Mater Res B Appl Biomater. 2009;91(2):930-7.

16- Lee SY, Huang HM, Lin $\mathrm{CY}$, Shih $\mathrm{Y}-\mathrm{H}$. Leached components from dental composites in oral simulating fluids and the resultant composite strengths. J Oral Rehabil. 1998;25(8):575-88.

17- Lopes LG, Franco EB, Pereira JC, Mondelli RF. Effect of lightcuring units and activation mode on polymerization shrinkage and shrinkage stress of composite resins. J Appl Oral Sci. 2008;16(1):35-42.

18- Lopes LG, Jardim Filho AV, Souza JB, Rabelo D, Franco EB, Freitas GC. Influence of pulse-delay curing on sorption and solubility of a composite resin. J Appl Oral Sci. 2009;17(1):27-31. 19- Moreira FCL, Antoniosi Filho NR, Souza JB, Lopes LG. Sorption, solubility and residual monomers of a dental adhesive cured by different light-curing units. Braz Dent J. 2010;21(5):432-8.

20- Nakayama WT, Hall DR, Grenoble DE, Katz JL. Elastic properties of dental resin restorative materials. J Dent Res. 1974;53(5):1121-6.

21- Obici AC, Sinhoreti MAC, Frollini E, Correr-Sobrinho L, Goes $M F$, Henriques GEP. Monomer conversion at different dental composite depths using six light-curing methods. Polymer Testing. 2006;25(3):282-8.

22- Park YJ, Chae KH, Rawls HR. Development of a new photoinitiation system for dental light-cure composite resins. Dent Mater. 1999;15:120-7.

23- Peutzfeldt A, Asmussen E. Resin composite properties and energy density of light cure. J Dent Res. 2005;84(7):659-62.

24- Porto IC, Soares LE, Martin AA, Cavalli V, Liporoni PC. Influence of the photoinitiator system and light photoactivation units on the degree of conversion of dental composites. Braz Oral Res. $2010 ; 24(4): 475-81$.

25- Rüttermann S, Dluzhevskaya I, Grosssteinbeck G, Raab WH, Janda R. Impact of replacing Bis-GMA and TEGDMA by other commercially available monomers on the properties of resin-based composites. Dent Mat. 2010;26(4):353-9.

26- United States Food and Drug Administration. Recommendations for chemistry data for indirect food additives petitions. Washington: FDA; 1988.

27- Wang L, D'Alpino PHP, Lopes LG, Pereira JC. Mechanical properties of dental restorative materials: relative contribution of laboratory tests. J Appl Oral Sci. 2003;11(3):162-7.

28- Witzel MF, Calheiros FC, Gonçalves F, Kawano Y, Braga RR. Influence of photoactivation method on conversion, mechanical properties, degradation in ethanol and contraction stress of resinbased materials. J Dent. 2005;33(9):773-9.

29- Yap AU, Soh MS, Han TT, Siow KS. Influence of curing lights and modes on cross-link density of dental composites. Oper Dent. 2004;29:410-5.

30- Yoshikawa T, Burrow MF, Tagami J. A light curing method for improving marginal sealing and cavity wall adaptation of resin composite restorations. Dent Mat. 2001;17(4):359-66. 